\title{
An Evolving Stellar Initial Mass Function and the Gamma-Ray Burst Redshift Distribution
}

\author{
F. Y. Wang ${ }^{1,2}$ and Z. G. Dai ${ }^{1,2}$ \\ ${ }^{1}$ Department of Astronomy, Nanjing University, Nanjing 210093, China \\ ${ }^{2}$ Key laboratory of Modern Astronomy and Astrophysics (Nanjing University), Ministry of \\ Education, Nanjing 210093, China
}

\begin{abstract}
Recent studies suggest that Swift gamma-ray bursts (GRBs) may not trace an ordinary star formation history. Here we show that the GRB rate turns out to be consistent with the star formation history with an evolving stellar initial mass function (IMF). We first show that the latest Swift sample of GRBs reveals an increasing evolution in the GRB rate relative to the ordinary star formation rate at high redshifts. We then assume only massive stars with masses greater than the critical value to produce GRBs, and use an evolving stellar IMF suggested by Davé (2010) to fit the latest GRB redshift distribution. This evolving IMF would increase the relative number of massive stars, which could lead to more GRB explosions at high redshifts. We find that the evolving IMF can well reproduce the observed redshift distribution of Swift GRBs.
\end{abstract}

Subject headings: cosmology: theory - gamma rays: bursts - stars: mass function

\section{Introduction}

Gamma-ray bursts (GRBs) are brief flashes of $\gamma$-rays occurring at an average detection rate of a few events per day at cosmological distances. Because of their very high luminosity, GRBs can be detected out to the edge of the visible Universe (Ciardi \& Loeb 2000; Lamb \& Reichart 2000; Bromm \& Loeb 2002; Gou et al. 2004). Thus, GRBs are ideal tools for probing the star formation rate, the reionization history, and the metal enrichment history of the Universe (Totani 1997; Campana et al. 2007; Bromm \& Loeb 2007). The advantages of GRBs over quasars for probing the high-redshift Universe had been discussed by Bromm \& Loeb (2007). In addition, GRBs have been used as standard candles to constrain cosmological parameters and dark energy (Dai, Liang \& Xu 2004; Friedman \& Bloom 2005; Wang \& Dai 2006; Schaefer 2007, and references therein). 
The association of long GRBs with core-collapse supernovae naturally suggests that the cosmic GRB rate should trace the star formation history. This gave rise to the expectation that GRBs may be a good tracer of cosmic star formation (Totani 1997; Wijers et al. 1998; Lamb \& Reichart 2000; Blain \& Natarajan 2000; Porciani \& Madau 2001). However, it was found that the rate of GRBs increases with cosmic redshift faster than the ordinary star formation rate (SFR) does (Daigne et al. 2006; Le \& Dermer 2007; Kistler et al. 2008, 2009; Yüksel \& Kistler 2007; Cen \& Fang 2007; Li 2008; Wang \& Dai 2009; Butler et al. 2010; Wanderman \& Piran 2010). The reason for this discrepancy has been unknown.

By investigating the redshift distribution of Swift GRBs, Guetta \& Piran (2007) found that the observed high-redshift bursts are more than the expectation from an ordinary star formation history ( $\mathrm{SFH}$ ) and thus the high-redshift GRB rate is inconsistent with the one inferred from the current model for the SFR. Furthermore, Kistler et al. (2008) found that the GRB rate at redshift $z \simeq 4$ is about four times larger than expected from star formation measurements. Daigne et al. (2006) concluded that GRB properties or progenitors must evolve with cosmic redshift to reconcile the observed GRB redshift distribution with the measured SFH. Li (2008) explained the observed discrepancy between the GRB rate history and the star formation rate history as being due to cosmic metallicity evolution, by assuming that long GRBs tend to occur in galaxies with low metallicities. However, very recently Levesque et al. (2010a,b) found several high-metallicity long GRB host environments, which suggests that a low-metallicity cut-off is unlikely (also see Graham et al. 2009). Xu \& Wei (2008) used a factitious stellar initial mass function (IMF) evolving with redshift to interpret the GRB redshift distribution. Cheng et al. (2010) suggested that this discrepancy could be eliminated if some high-redshift GRBs are ascribed to electromagnetic bursts of superconducting cosmic strings, although the existence of the superconducting cosmic strings has remained controversial.

In this Letter, we first enlarge the GRB sample with 122 long GRBs observed by Swift. Then we interpret the latest Swift GRB redshift distribution using a reasonable evolving stellar initial mass function (IMF) proposed by Davé (2010). The structure of this paper is as follows: in section 2, we give an evolving initial mass function form, and in section 3, we show the analysis method. The results are presented in section 4 and conclusions are shown in section 5 .

\section{An evolving initial mass function}

The ordinary form of stellar IMF proposed by Salpeter (1955) is

$$
d N=m^{-1.35} d \log m .
$$


It was shown that the high-redshift GRB rate exceeds the expectation based on the above SFR. This leads us to invoking a different form of stellar IMF. The possibility of an evolving IMF was discussed several times in the literature (e.g. Larson 1998, 2005; Ferguson, Dickinson \& Papovich 2002; Fardal et al. 2007; van Dokkum 2008). Kroupa (2001) pointed out that a universal IMF is not expected theoretically, though no variations had been unequivocally detected in the studies of local star-forming regions. Scalo (1998) mentioned that although the IMF index may vary at different redshifts in the Universe, its average value is close to the Salpeter value. Wilkins, Trentham \& Hopkins (2008) independently determined the cosmic stellar mass growth rate by compiling observations of stellar mass densities from the literature and suggested an evolving IMF to interpret the discrepancy between the stellar mass density and SFH. In order to reconcile the discrepancy between the theory predicting the galaxy stellar mass-SFR relation with the observations, Davé (2008) proposed an evolving IMF with the following form

$$
\frac{d N}{d \log m}=\xi(m) \propto \begin{cases}m^{-0.3} \text { for } m<\hat{m}_{\mathrm{IMF}} \\ m^{-1.3} \text { for } m>\hat{m}_{\mathrm{IMF}}\end{cases}
$$

where $\hat{m}_{\mathrm{IMF}}=0.5(1+z)^{2} M_{\odot}$, which has been constrained by requiring non-evolving star formation activity parameter. It is worth noting that this evolving IMF is only constrained out to $z \sim 2$ from the galaxy stellar mass-SFR relation, though its predictions are consistent with the other observations out to $z \sim 4$. Chary (2008) found that this IMF would produce sufficient ionizing photons to account for late reionization of the intergalactic medium if it evolved out to $z>4$. So the IMF suggested by Davé (2008) can be used to $z>4$. More recently, Davé (2010) found $\hat{m}_{\mathrm{IMF}}=0.5(1+z)^{3-0.75 z} M_{\odot}$ using the Herschel data.

\section{The method}

We consider a spatial volume $V$ at redshift $z$. The IMF can be written as $A \xi(m)$, so $\int_{m_{s}}^{m_{l}} A m \xi(m) d \log m=R_{\mathrm{SFR}} V$, where $R_{\mathrm{SFR}}$ is the SFR, $m_{l}$ is the largest mass of stars and $m_{s}$ is the smallest mass of stars. We consider only massive stars with masses larger than $30 M_{\odot}$ can produce GRBs (Woosley 1993; Bissaldi et al. 2007)1, so

$$
R_{\mathrm{GRB}} \propto \frac{N_{m>30 M_{\odot}}}{V}=K\left(\frac{c}{H_{0}}\right)^{-3} \frac{\int_{30 M_{\odot}}^{m_{l}} \xi(m) d \log m}{\int_{m_{s}}^{m_{l}} m \xi(m) d \log m} R_{\mathrm{SFR}}
$$

\footnotetext{
${ }^{1}$ The lower limit mass of a star that can collapse to GRB is uncertain at present. But this value is unimportant in our analysis below. The best fitting parameters will shift slightly when the lower limit mass is changed. But this evolving IMF could still interpret the GRB redshift distribution.
} 
where $K$ is a constant to be constrained and $R_{\mathrm{GRB}}$ is the rate of GRBs, representing the number of GRBs per unit time per unit volume at redshift $z$. We use the SFR derived by Hopkins \& Beacom (2006),

$$
\log R_{\mathrm{SFR}}(z)=a+b \log (1+z)
$$

with

$$
(a, b)= \begin{cases}(-1.70,3.30), & z<0.993 \\ (-0.727,0.0549), & 0.993<z<3.80 \\ (2.35,-4.46), & z>3.80\end{cases}
$$

Le Borgne et al. (2009) used mid- and far-infrared observations to constrain the SFR and found that $\mathrm{SFH}$ is well-constrained and consistent with direct measurements from Hopkins \& Beacom (2006). So we also use the results of Hopkins \& Beacom (2006).

Then the observed rate of GRBs within $z \sim z+d z$ and $L \sim L+d L$ is

$$
\frac{d N}{d t}=\Phi(L) \frac{R_{\mathrm{GRB}}}{1+z} \frac{\Delta \Omega_{s}}{4 \pi} \frac{d V_{\mathrm{com}}(z)}{d z} d L d z
$$

where $\Phi(L)$ is the beaming-convolved luminosity function of GRBs, $(1+z)^{-1}$ is due to cosmological time dilation and $\Delta \Omega_{s}=1.4 \mathrm{sr}$ is the solid angle covered on the sky by Swift (Salvaterra \& Chincarini 2007). In a flat universe, the comoving volume is calculated by

$$
\frac{d V_{\mathrm{com}}}{d z}=4 \pi D_{\mathrm{com}}^{2} \frac{d D_{\mathrm{com}}}{d z}
$$

where the comoving distance is

$$
D_{\mathrm{com}}(z) \equiv \frac{c}{H_{0}} \int_{0}^{z} \frac{d z^{\prime}}{\sqrt{\Omega_{m}\left(1+z^{\prime}\right)^{3}+\Omega_{\Lambda}}} .
$$

In the calculations, we use $\Omega_{m}=0.3, \Omega_{\Lambda}=0.7$ and $H_{0}=70 \mathrm{~km} \mathrm{~s}^{-1} \mathrm{Mpc}^{-1}$. There are many luminosity function forms in the literature. We use the Schechter-function form

$$
\Phi(L)=\frac{1}{L_{\star}}\left(\frac{L}{L_{\star}}\right)^{\beta} \exp \left(-L / L_{\star}\right),
$$

where $\beta$ and $L_{\star}$ are constant parameters to be determined by the observational data. The observed distribution of $L_{\text {iso }}$ is then given by

$$
\Delta N(L)=\Phi(L)\left[\int_{0}^{z_{\max }(L)} \frac{R_{\mathrm{GRB}}(z)}{1+z} \frac{\Delta \Omega_{s}}{4 \pi} \frac{d V_{\mathrm{com}}}{d z} d z\right] \Delta L \Delta t_{\mathrm{obs}}
$$


where $z_{\max }=z_{\max }\left(L_{\text {iso }}\right)$ is the maximum redshift up to which a GRB with luminosity $L_{\text {iso }}$ can be detected by Swift, solved from equation $L_{\text {lim }}(z)=L_{\text {iso }}$.

The isotropic-equivalent luminosity of a GRB can be obtained by $L_{\text {iso }}=E_{\text {iso }}(1+z) / T_{90}$ (Kistler et al. 2008). We use 122 long GRBs observed by Swift2 till GRB 090726 (Butler et al. 2010). The distribution of $L_{\text {iso }}$ for the 122 GRBs in the sample is shown in Fig.1. The luminosity threshold can be approximated by a bolometric energy flux limit $F_{\text {lim }}=$ $1.2 \times 10^{-8} \mathrm{erg} \mathrm{cm}^{-2} \mathrm{~s}^{-1}$. The luminosity threshold is then

$$
L_{\lim }=4 \pi D_{L}^{2} F_{\lim },
$$

where $D_{L}$ is the luminosity distance to the burst.

With the above luminosity threshold and an adopted GRB rate history, the observed luminosity distribution can be fitted by an intrinsic Schechter luminosity function with a power-law index $\beta=-1.12$, a characteristic luminosity $L_{\star}=9.16 \times 10^{52} \mathrm{erg} \mathrm{s}^{-1}$ and $\Delta t_{\text {obs }} K=69858.51$ with $\chi_{r}^{2}=1.15$.

\section{The results}

In order to study the rates of GRBs and star formation, it is convenient to use a dimensionless $Q$, where $Q=Q(z)$ is defined by (Kistler et al. 2007)

$$
Q(z) \equiv\left(\frac{c}{H_{0}}\right)^{-3} \int_{0}^{z} \frac{1}{1+z^{\prime}} \frac{d V_{\mathrm{com}}}{d z^{\prime}} d z^{\prime} .
$$

The coordinate $Q$ is particularly useful in binning the data, as the definition of $Q$ has taken into account both the effect of the comoving volume and the effect of cosmic time dilation. For example, when the comoving rate density of GRBs was a constant, in each equally sized bin of $Q$, the observed GRBs number would be a constant. The complete GRB selection function is very difficult to determine (Coward 2007). We choose the GRBs in the cuts $L_{\text {iso }}>0.8 \times 10^{51} \mathrm{erg} \mathrm{s}^{-1}$ and in the redshift range $0-4$ (Kistler et al. 2008). This method can reduce the selection effect by removing many low- $z$, low- $L_{\text {iso }}$ bursts that could not have been seen at higher redshifts. There are $72 \mathrm{GRBs}$ in this sample. The SFR fit from Hopkins \& Beacom (2006) in this range is shown as the dotted line in Fig. 2. We can see the GRB rate is incompatible with the expectation from the ordinary SFR. The solid line shows the cumulative distribution of the 72 Swift GRBs. The result from an evolving IMF suggested by Davé (2010) is shown as the solid line, which agrees with the observed data very well.

\footnotetext{
${ }^{2}$ See http://swift.gsfc.nasa.gov/docs/swift/archive/grb_table
} 
From Eqs (8), (11) and (14), we can obtain the observed number of GRBs in an observer's time interval $\Delta t_{\mathrm{obs}}$, and with $Q$ in the interval $Q-(Q+d Q)$,

$$
\Delta N(Q)=\left[\int_{L_{\mathrm{lim}}(z)}^{\infty} \Phi(L) \frac{R_{\mathrm{GRB}}(z)}{1+z} \frac{\Delta \Omega_{s}}{4 \pi} \frac{d V(z)}{d z} \frac{d z}{d Q} d L\right] d Q \Delta t_{\mathrm{obs}}
$$

where $L_{\lim }(z)$ is determined by Eq. (11).

We use the luminosity cut $L_{\lim }=0.8 \times 10^{51} \mathrm{erg} \mathrm{s}^{-1}$ (Li 2008). The cut in luminosity and redshift minimizes the selection effect in the GRB data. The total number of GRBs with $L_{\text {iso }}>L_{\text {lim }}$ is 72 . The distribution of $Q$ for the 72 Swift GRBs is plotted in Fig. 3. Because of the flux limit of the detector (Kistler et al. 2008), the model deviates from the data at $z>4$. The solid line shows the best fit of the $R_{\mathrm{GRB}}$ to the first six data points using an evolving IMF. The dashed line shows the best fit from non-evolving IMF.

Fig. 4 shows the distribution of $Q$ for all 122 Swift GRBs in the sample. The solid line is $N(Q)$ calculated by Eq. 13 with the normalization and the luminosity function parameters

determined above, and $L_{\text {lim }}$ calculated by Eq. 11. We can see that the modeled $N(Q)$ fits the observational data very well with $\chi_{r}^{2}=1.14$. However, there is an obvious excess in the number of GRBs in the bin of $0<Q<1$, which might be caused by statistical fluctuations. If we exclude it, the $\chi_{r}^{2}$ decreases to 0.32 .

\section{Conclusions}

In this Letter, we have presented that the redshift distribution of Swift GRBs with measured redshifts and calculated luminosities can be successfully fitted by the SFH with an evolving stellar IMF. It is widely considered by current theories that only massive stars with masses larger than the critical value can produce long GRBs. The evolving stellar IMF becoming increasingly top heavy at larger z suggested by Davé (2010) can lead to more GRBs produced at high redshifts.

Kistler et al. (2008) considered several possible reasons for the discrepancy between the Swift GRB rate and the SFH. They showed that the Kolmogorov-Smirnov test does not favor an interpretation as a statistical anomaly. Selection effects are also unlikely to cause an increased efficiency in detecting high-redshift GRBs. Although Kistler et al. (2008) have argued that alternative reasons are possible (e.g., evolution in the fraction of binary systems, an evolving IMF of stars, cosmic metallicity evolution), they did not give a quantitative analysis or a detailed discussion of the evolving IMF. We enlarged the GRB sample with 122 long GRBs and used a reasonable evolving IMF. The results in this paper indicate that the evolving IMF may explain the redshift distribution of Swift GRBs. If the redshift 
distribution of GRBs and SFH are well measured, GRBs would be used to probe the stellar IMF at high redshifts.

We thank D. M. Wei for valuable discussions and an anonymous referee for useful suggestions and comments. This work is supported by the National Natural Science Foundation

of China (grants 10873009 and 11033002) and the National Basic Research Program of China (973 program) No. 2007CB815404.

\section{REFERENCES}

Bissaldi, E., Calura, F., Matteucci, F., Longo, F., \& Barbiellini, G. 2007, A\&A, 471, 585

Blain, A. W. \& Natarajan, P. 2000, MNRAS, 312, L35

Bromm, V. \& Loeb, A. 2002, ApJ, 575, 111

—. 2007, arXiv:0706.2445v2

Butler, N. R., Bloom, J. S., \& Poznanski, D. 2010, ApJ, 711, 495

Campana, S., Lazzati, D., Ripamonti, E., Perna, R., Covino, S., Tagliaferri, G., Moretti, A., Romano, P., Cusumano, G., \& Chincarini, G. 2007, ApJ, 654, L17

Cen, R. \& Fang, T. 2007, arXiv: 0710.4370v1

Chary, R. 2008, ApJ, 680, 32

Cheng, K. S., Yu, Y., \& Harko, T. 2010, Physical Review Letters, 104, 241102

Ciardi, B. \& Loeb, A. 2000, ApJ, 540, 687

Coward, D. 2007, New A Rev., 51, 539

Dai, Z. G., Liang, E. W., \& Xu, D. 2004, ApJ, 612, L101

Daigne, F., Rossi, E. M., \& Mochkovitch, R. 2006, MNRAS, 372, 1034

Davé, R. 2008, MNRAS, 385, 147

—. 2010, arXiv:1008.5283

Fardal, M. A., Katz, N., Weinberg, D. H., \& Davé, R. 2007, MNRAS, 379, 985 
Ferguson, H. C., Dickinson, M., \& Papovich, C. 2002, ApJ, 569, L65

Friedman, A. S. \& Bloom, J. S. 2005, ApJ, 627, 1

Gou, L. J., Mészáros, P., Abel, T., \& Zhang, B. 2004, ApJ, 604, 508

Graham, J. F., Fruchter, A. S., Kewley, L. J., Levesque, E. M., Levan, A. J., Tanvir, N. R., Reichart, D. E., \& Nysewander, M. 2009, in American Institute of Physics Conference Series, Vol. 1133, American Institute of Physics Conference Series, ed. C. Meegan, C. Kouveliotou, \& N. Gehrels, 269-272

Guetta, D. \& Piran, T. 2007, J. Cosmology Astropart. Phys., 7, 3

Hopkins, A. M. \& Beacom, J. F. 2006, ApJ, 651, 142

Kistler, M. D., Yüksel, H., Beacom, J. F., Hopkins, A. M., \& Wyithe, J. S. B. 2009, ApJ, 705, L104

Kistler, M. D., Yüksel, H., Beacom, J. F., \& Stanek, K. Z. 2008, ApJ, 673, L119

Kroupa, P. 2001, MNRAS, 322, 231

Lamb, D. Q. \& Reichart, D. E. 2000, ApJ, 536, 1

Larson, R. B. 1998, MNRAS, 301, 569

-. 2005, MNRAS, 359, 211

Le, T. \& Dermer, C. D. 2007, ApJ, 661, 394

Le Borgne, D., Elbaz, D., Ocvirk, P., \& Pichon, C. 2009, A\&A, 504, 727

Levesque, E. M., Kewley, L. J., Graham, J. F., \& Fruchter, A. S. 2010a, ApJ, 712, L26

Levesque, E. M., Soderberg, A. M., Kewley, L. J., \& Berger, E. 2010b, 2010b, ApJ, 725, 1337

Li, L. 2008, MNRAS, 388, 1487

Porciani, C. \& Madau, P. 2001, ApJ, 548, 522

Salpeter, E. E. 1955, ApJ, 121, 161

Salvaterra, R. \& Chincarini, G. 2007, ApJ, 656, L49 
Scalo, J. 1998, in Astronomical Society of the Pacific Conference Series, Vol. 142, The Stellar Initial Mass Function (38th Herstmonceux Conference), ed. G. Gilmore \& D. Howell, 201

Schaefer, B. E. 2007, ApJ, 660, 16

Totani, T. 1997, ApJ, 486, L71

van Dokkum, P. G. 2008, ApJ, 674, 29

Wanderman, D. \& Piran, T. 2010, MNRAS, 406, 1944

Wang, F. Y. \& Dai, Z. G. 2006, MNRAS, 368, 371

-. 2009, MNRAS, 400, L10

Wijers, R. A. M. J., Bloom, J. S., Bagla, J. S., \& Natarajan, P. 1998, MNRAS, 294, L13

Wilkins, S. M., Trentham, N., \& Hopkins, A. M. 2008, MNRAS, 385, 687

Woosley, S. E. 1993, ApJ, 405, 273

Xu, C. Y. \& Wei, D. M. 2008, Acta Astronomica Sinica, 49, 387

Yüksel, H. \& Kistler, M. D. 2007, Phys. Rev. D, 75, 083004 


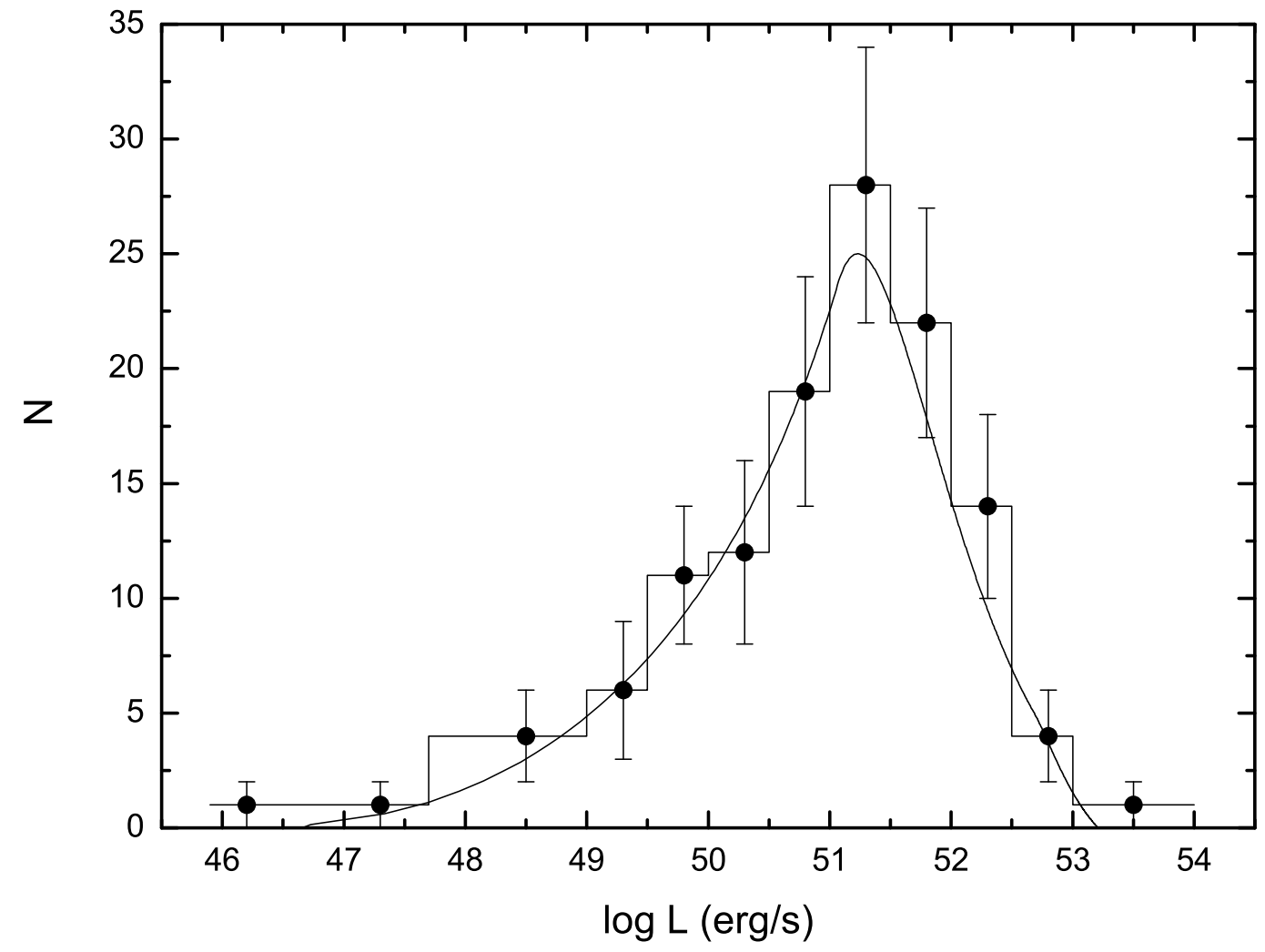

Fig. 1.- Distribution of the isotropic-equivalent luminosity for 122 long-duration Swift GRBs. The solid line is plotted according to Eq. 12 using best fitted parameters. 


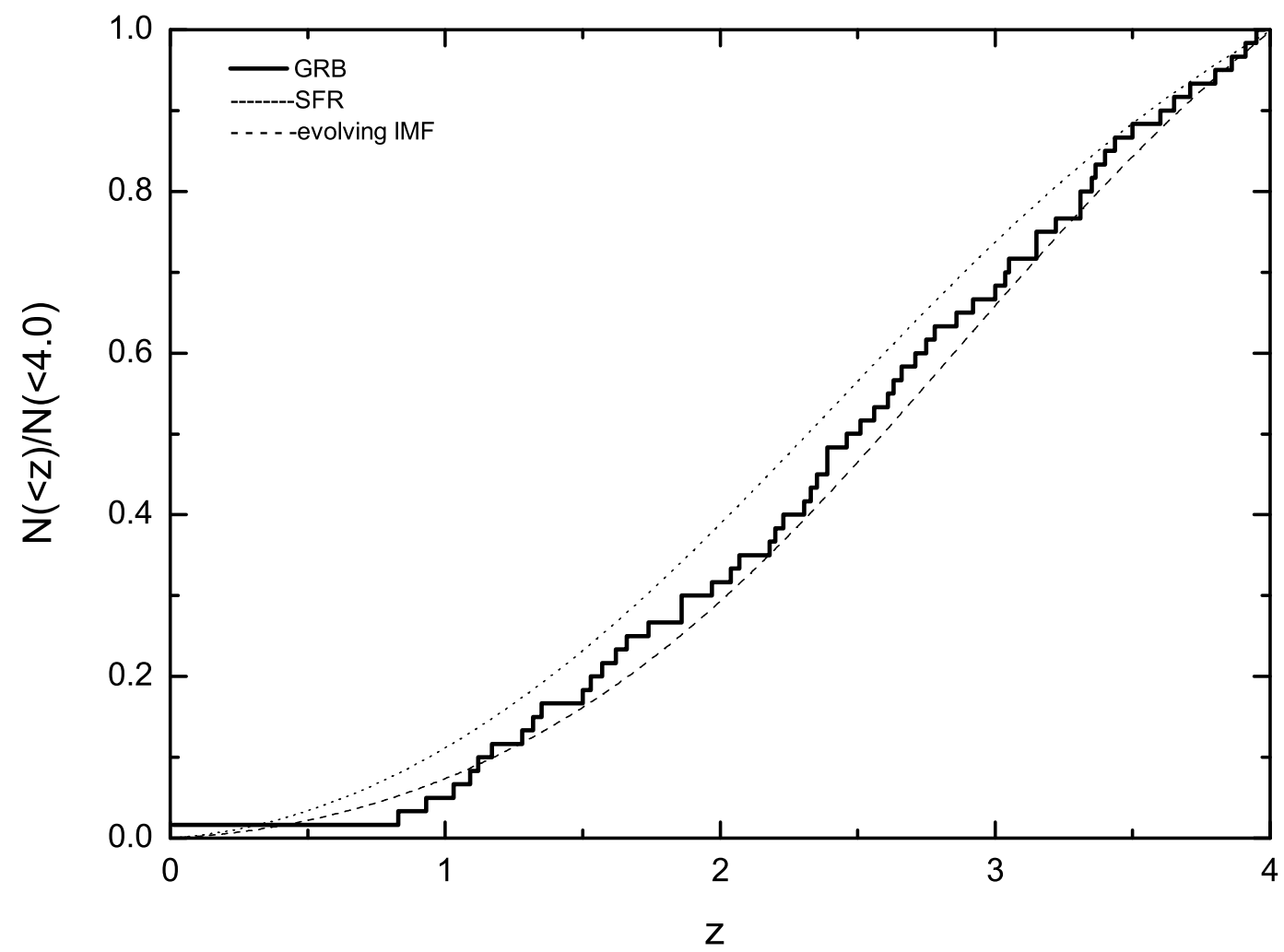

Fig. 2.- The cumulative distribution of 72 Swift long GRBs with $L_{\text {iso }}>0.8 \times 10^{51} \mathrm{erg} \mathrm{s}^{-1}$ (stepwise solid line). The dotted line shows the GRB rate inferred from the star formation history of Hopkins \& Beacom (2006). The dashed line shows the GRB rate inferred from star formation history including an evolving IMF. 


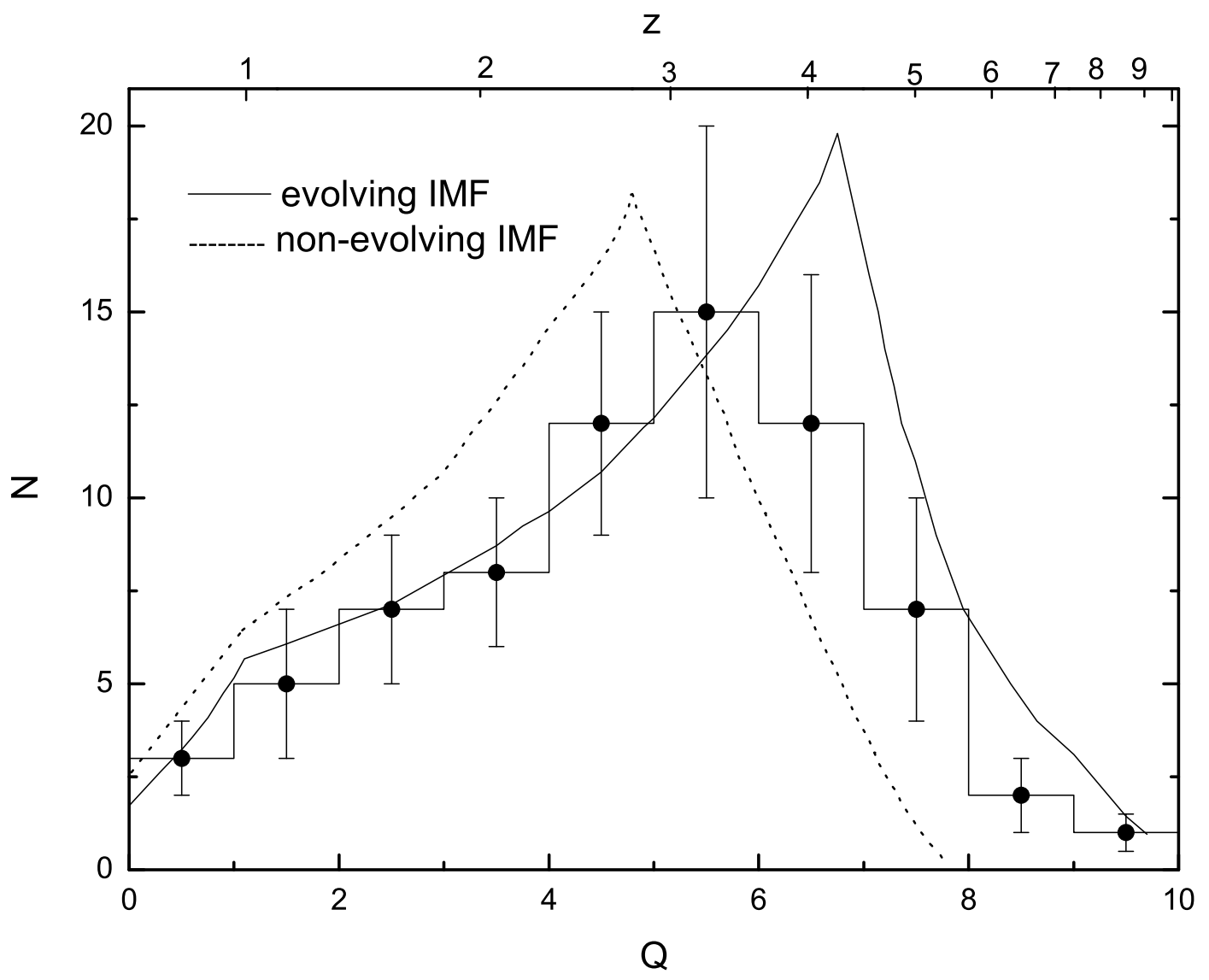

Fig. 3.- Distribution of $Q$ for 72 Swift GRBs with $L_{\text {iso }}>0.8 \times 10^{51} \mathrm{erg} \mathrm{s}^{-1}$ (the solid histogram, with the number of GRBs in each bin indicated by a dark point with Poisson error bars). The solid line is the best fit of the GRB rate. The dotted curve shows the best fit by non-evolving IMF. 


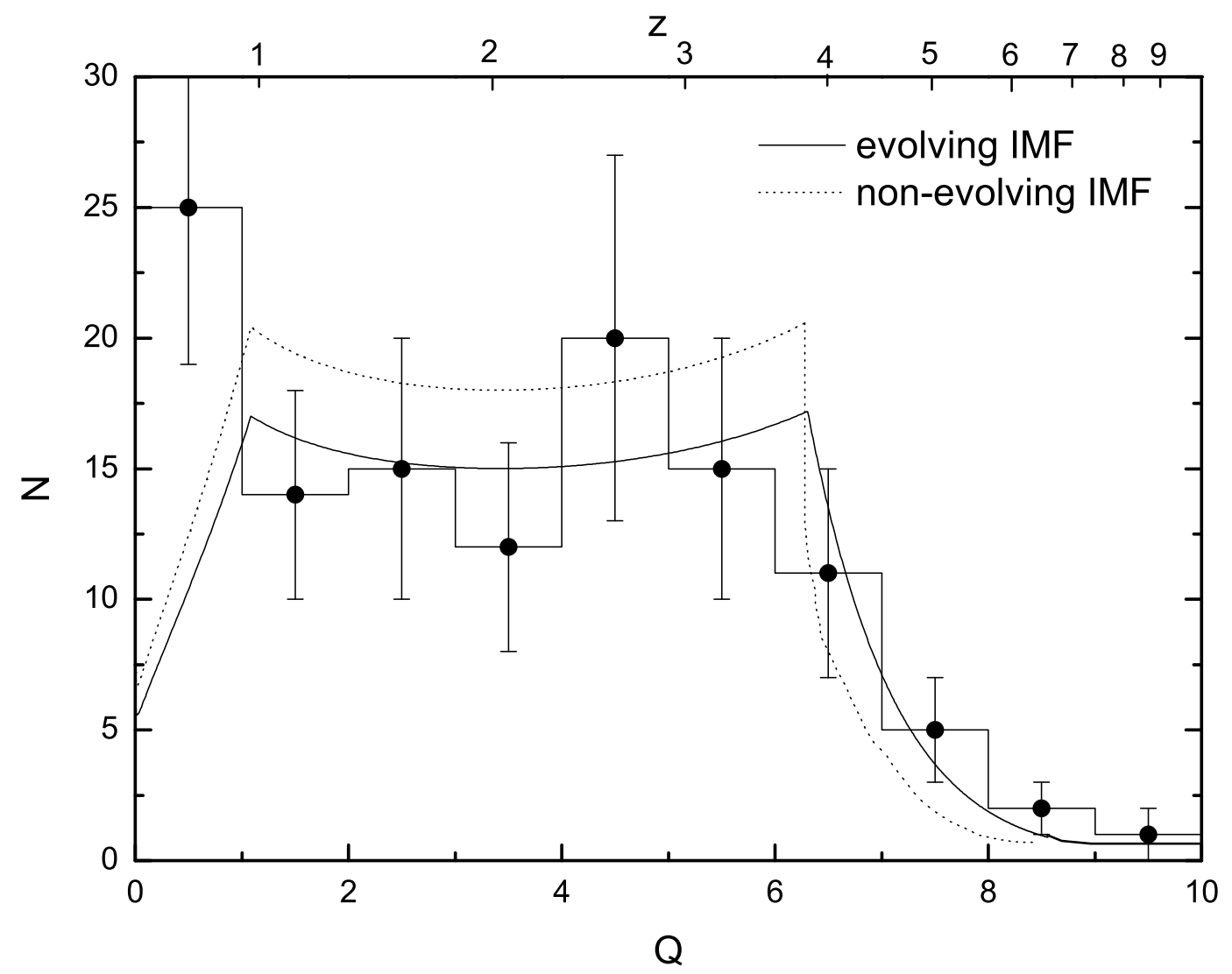

Fig. 4.- Distribution of $Q$ for all the 122 Swift GRBs, the points with error bars represent the number of GRBs lying between $Q_{i} \sim Q_{i}+1$. The solid curve shows the best fit by Eq. 13. The dotted curve shows the best fit by non-evolving IMF. 